\title{
LA MOTIVACIÓN Y EL ESTILO DE APRENDIZAJE REFLEXIVO- CRÍTICO EN LA UNIVERSIDAD NACIONAL DE INGENIERÍA MOTIVATION AND LEARNING STYLE REFLECTIVE-CRITICAL IN THE UNIVERSIDAD NACIONAL DE INGENIERÍA
}

\author{
Romulo Romero Centeno ${ }^{1}$
}

\begin{abstract}
RESUMEN
La presente investigación tuvo como objetivo fundamental establecer el grado de relación existente entre la motivación intrínseca y el estilo de aprendizaje reflexivo-crítico en los estudiantes de la Universidad Nacional de Ingeniería (UNI), durante el año académico 2009 II. La muestra de estudio estuvo conformada por 371 universitarios, de sexo masculino y femenino, de condición económica media y medio baja, de condición académica regular, cuyas edades varían de 16 a 32 años, de las distintas facultades de la Universidad Nacional de Ingeniería. La muestra fue seleccionada en forma probabilística mediante el proceso del azar simple. El diseño de investigación adoptado fue el correlacional para establecer la relación entre la motivación intrínseca y estilo de aprendizaje reflexivo-crítico en los estudiantes de ingeniería. Para identificar el nivel de motivación intrínseca se utilizó el test de motivación intrínseca. El nivel de desarrollo del estilo de aprendizaje reflexivo-crítico fue medido por un cuestionario de estilos de aprendizaje reflexivo crítico. Los resultados muestran que (1) el nivel de la motivación intrínseca alcanzado por los estudiantes universitarios de la Universidad Nacional de Ingeniería se ubica en el nivel alto y medio. (2) El nivel de desarrollo del Estilo de aprendizaje reflexivo-crítico en los estudiantes de la Universidad de Ingeniería fue alto y medio; y (3) respecto a la correlación entre el nivel de motivación intrínseca y el nivel de desarrollo del estilo de aprendizaje reflexivocrítico se encontró una relación significativa. Se concluye que, en la muestra estudiada, existen relaciones significativas entre la motivación intrínseca y el estilo de aprendizaje reflexivo-crítico en los estudiantes universitarios de la Universidad Nacional de Ingeniería.
\end{abstract}

Palabras claves.- La motivación intrínseca - Estilo de aprendizaje reflexivo-Crítico - Estudiantes de la Universidad Nacional de Ingeniería.

\begin{abstract}
This investigation had as a fundamental aim to establish the relationship between the intrinsic motivation and the learning style reflective-critical of the students from the Universidad Nacional de Ingeniería during the academic year 2009 II. The study sample was consisted of 371 college students, male and female, economically disadvantaged middle and lower middle regular academic status whose ages range from 16 to 32 years, and also students from the different faculties of the Universidad Nacional de Ingeniería. The sample was selected in a probabilistic way by simple random process. The research design adopted was the correlation to establish the relationship between intrinsic motivation and learning style reflective-critical engineering students. The intrinsic motivation test was used to identify the level of the intrinsic motivation of
\end{abstract}

\footnotetext{
${ }^{1}$ Ing. Docente de la Facultad de Ingeniería Ambiental de la Universidad Nacional de Ingeniería.
} 
the students while the level of development of the style-critical reflective learning was measured by a questionnaire of critical reflective learning styles. The results show that (1) the level of intrinsic motivation achieved by university students of different faculties of the Universidad Nacional de Ingeniería is located in the high and medium level. (2) The level of development of reflective learning style-critical students of different faculties of the University of Engineering was high and medium, and (3) regarding the correlation between the level of intrinsic motivation and level of development learning style-critical reflective found a significant relationship. So we may conclude in this study sample that there are significant relationships between the intrinsic motivation and the learning style, critical reflective college students at the Universidad Nacional de Ingeniería.

Key words.- Intrinsic motivation - Learning style-critical and thoughtful students of the Universidad Nacional de Ingeniería.

\section{INTRODUCCIÓN}

La búsqueda de la excelencia en las universidades tiene entre los resultados la calidad de sus graduados. Formar un estudiante con calidad significa capacitarlo no sólo para desenvolverse en el presente, sino para que en el futuro tenga capacidad de decisión en los diferentes ámbitos de su vida (personal, familiar, laboral, socio-cultural). La educación de calidad se logra con una educación formal sistemática. En esta formación de calidad incide, en la institución formadora, el empleo de adecuadas estrategias motivacionales y de apropiados estilos de aprendizaje. Si bien los estudiantes asumen diferentes tipos y niveles de motivación, de estilos de aprendizaje, distintas actitudes acerca de la enseñanza y del aprendizaje, diversas respuestas en ambientes de aprendizaje y prácticas instruccionales específicas, las estrategias de enseñanza-aprendizaje, para que sean efectivas, deberían seleccionarse considerando esta diversidad de estilos.

Un objetivo fundamental de la educación superior consiste en aprender a aprender. Para ello, el estudiante debe sentirse motivado intrínsecamente (que la motivación parta de él) y ejercite un estilo de aprendizaje reflexivo-crítico. El aprender a aprender, permite ser más activo, reflexivo e independiente, garantizando su autonomía o autocontrol en este proceso. Enseñar a aprender a pensar permitirá en el alumno el desarrollo de un estilo de aprendizaje reflexivo-crítico.
En la universidad, la mayoría de los estudiantes no preguntan en clase por falta de confianza. Sin clima motivacional favorable y sin la práctica de un estilo de aprendizaje reflexivo-crítico, se acentúan los aprendizajes repetitivos y mecánicos, con una clara incidencia negativa sobre la comprensión. Sin embargo, cuando se emprenden tareas apelando a una motivación intrínseca y con un estilo de aprendizaje reflexivo-crítico, no se necesita superar ningún preconcepto respecto a aburrimiento, temor al fracaso, aparente incompetencia para la tarea. Si al estudiar se tiene expectativas claras y positivas, si se piensa en el logro futuro, resultan gratificantes las tareas a emprenderse.

Hay, en este sentido, mucho que trabajar con los docentes universitarios. Ellos deberán generar esa motivación intrínseca, ese deseo de trabajar identificado con el tema o materia, con la carrera o especialidad. Sin la motivación intrínseca, los estudiantes apelan más a una motivación extrínseca, por lo que sus metas e intereses no los inducen a poner en juego el esfuerzo y las estrategias adecuadas para aprender. Una motivación extrínseca viene de afuera, no sale de él. Por ejemplo, estudian porque hay mayor rentabilidad, pero no están identificados con su actividad. Resulta necesario asegurarse de que los estudiantes estén motivados a la hora de plantearles objetivos, retos y actividades. El hacer algo obligado no pone en juego la motivación intrínseca; por el contrario, destruye el interés y los 
logros son menores ya que se busca salir de esa situación como sea. Asimismo, la importancia de la reflexión-crítica radica en la evaluación concienzuda, en la búsqueda de los pros y contras de las acciones, de los proyectos que permitan el beneficio de las mayorías y sin el deterioro de nuestro hábitat.

Nickerson (1994) [1] manifiesta que gran parte del problema de aprender a pensar, y del pensamiento, es también un problema de motivación o un problema de estilo de aprendizaje. Pensar puede ser un trabajo exigente $\mathrm{y}$, sin duda, la principal razón de por qué las personas no lo hacen más, es por falta de motivación adecuada para realizar el esfuerzo o por no emplear el estilo de aprendizaje adecuado.

Las universidades tienen entre sus misiones formar estudiantes reflexivos, críticos y creativos. Para tal efecto, es necesario promover el aprendizaje crítico-reflexivo, mediante el cual el estudiante asume y dirige su potencial. Esta se perfecciona con la motivación intrínseca, que, a su vez, se potencia, a través del diálogo reflexivo, discusión controversial, investigación, debate, seminario, círculos de reflexión, etc.

La presente investigación parte de la preocupación de docentes universitarios y estudiantes de la Universidad Nacional de Ingeniería por formar mejor a los futuros ingenieros. Para ello, se debe promover la motivación intrínseca, como factor indispensable para el desarrollo del estilo de aprendizaje reflexivo-crítico, para facilitar aprendizajes significativos; por ende, para contribuir al mejoramiento de la calidad de la formación científica y la formación profesional de los estudiantes de ingeniería.

Roces Montero, González, Núñez y Álvarez (2000) [2] investigaron las relaciones entre motivación, estrategias de aprendizaje y rendimiento académico en estudiantes universitarios en la Universidad de Oviedo. Ellos concluyen que una motivación adecuada influye en el rendimiento indirectamente, por una implicación cognitiva del alumnado.
Bali Chaves, Cazares Castillo y Wisniewski (2000) [3] analizan y miden la motivación intrínseca como factor adecuado para alcanzar un aprendizaje permanente, enriquecedor, creativo y aplicado a la toma de decisiones y a la solución de problemas. En la muestra estudiada constataron los niveles moderadamente altos tanto en motivación intrínseca (saber) como de motivación extrínseca (regulación externa).

López García (2000) [4] investigó los estilos de aprendizaje y los estilos de enseñanza, un modelo de categorización de estilos de aprendizaje de los alumnos de enseñanza secundaria desde el punto de vista del profesor. La investigación concluye que el profesorado de enseñanza secundaria, desde una óptica práctica y funcional, considera útiles estas categorías de estilos de aprendizaje.

Camarero Suárez, Martín del Buey y Herrero Diez (2000) [5] investigaron los estilos y estrategias de aprendizaje en estudiantes universitarios. En cuanto a los estilos, confirma lo obtenido en investigaciones anteriores, que el estilo activo se encuentra preferentemente en carreras de humanidades, mientras que en el resto de los estilos (reflexivo. teórico, pragmático), los resultados no son coincidentes, lo que puede ser indicador de su independencia con la naturaleza de los estudios que se realizan.

Guanipa y Mogollón (2006) [6] investigaron los estilos de aprendizaje y estrategias cognitivas en estudiantes de ingeniería. Se destaca que un grupo experimental haya generado cuatro estrategias de aprendizaje. Demuestra que cuando el alumno presenta un estilo cognitivo predominante y es estimulado por estrategias de enseñanza adecuada, este profundizará en la tarea, actividad o trabajo académico asignado, logrando construir su conocimiento y mejorar su rendimiento académico. Esto le permite incrementar su capacidad de regulación y controlar su propio proceso de aprendizaje y así tener una mayor autonomía y alta autoestima.

Castro Gonzales (2000) [7] concluye que no hay estilos de aprendizaje definidos, notándose la 
presencia de los estilos de aprendizaje combinados en los que predomina el estilo reflexivo divergente en un $52 \%$ del total.

Delgado Vásquez (2004) [8] encontró que en el total de participantes maestristas, el estilo de aprendizaje predominante es el divergente, mientras que en los maestristas de la Universidad Nacional Mayor de San Marcos predomina el estilo asimilador y en los alumnos de Universidad Ricardo Palma predominan los estilos acomodador y divergente.

Huauya Quispe, Pedro (2008) [9] afirma que existe mayor desarrollo de los estilos de aprendizaje teórico y activo, siendo menor el reflexivo y pragmático, en estudiantes de secundaria de Ayacucho - Perú.

Palomino Orizano, Juan (2009) [10] confirma que existe correlación positiva fuerte entre las variables motivación, estilos cognoscitivos, estrategias de aprendizaje y la variable actividad personal.

Esta investigación apuesta al mejoramiento de la calidad en la formación integral del estudiante en la Universidad Nacional de Ingeniería. Para ello, promueve elevar la pertinencia, la calidad y la eficacia del proceso de enseñanza-aprendizaje en las distintas áreas de ingeniería. Esto, a su vez, eleva los niveles de calidad del desempeño profesional del ingeniero.

Resulta importante esta investigación por las razones siguientes: No existen en nuestro medio académico (Lima - Perú) investigaciones sobre la motivación intrínseca y su relación con el desarrollo del estilo de aprendizaje reflexivocrítico en los estudiantes de la Universidad Nacional de Ingeniería. Hay la necesidad de destacar la importancia de la motivación intrínseca como factor primordial para promover el desarrollo efectivo del estilo de aprendizaje reflexivo-crítico en los estudiantes de ingeniería.

El docente debe conocer el perfil de aprendizaje de cada alumno para adoptar el estilo de enseñanza a cada alumno (en lo posible) y conseguir una interacción efectiva.

El objetivo de la investigación consistió en determinar el grado de relación existente entre la motivación intrínseca y el estilo de aprendizaje reflexivo-crítico en los universitarios de la Universidad Nacional de Ingeniería, durante el segundo semestre académico 2009.

Una de las principales limitaciones metodológicas radicó en el diseño metodológico adoptado; ya que esto no está orientado a establecer una relación de causa y efecto. Los resultados obtenidos tienen validez y aplicabilidad en la Universidad Nacional de Ingeniería, Perú.

\section{METODOLOGÍA}

Materiales: La investigación, desarrollada en la Universidad Nacional de Ingeniería, se inició en setiembre del 2009 y se concluyó en 2012. Las condiciones de investigación fueron en un clima habitual, sin modificar el contexto. El trabajo abarca una población aproximada de 10,100 estudiantes entre varones y mujeres de distintas edades. El objeto de estudio fue determinar el grado de relación existente entre la motivación intrínseca y el estilo de aprendizaje reflexivocrítico en los estudiantes de la Universidad Nacional de Ingeniería, durante el segundo semestre académico 2009.

Método.- Para los efectos de la investigación, se tomó una muestra aleatoria simple, constituida por 371 estudiantes, de ambos sexos, de condición socioeconómica media y media baja, de situación académica regular, cuyas edades varían de 16 a 32 años, de la Universidad Nacional de Ingeniería.

Se utilizó el test de motivación intrínseca constituido de 31 ítems que se elaboró en base a los aportes de la teoría de la evaluación cognitiva y la teoría de la autodeterminación (Ryan y Deci; 2000) [11]. Tuvo el propósito de identificar el nivel de motivación intrínseca en los estudiantes de la Universidad Nacional de Ingeniería. Para la construcción del instrumento se empleó una matriz 
de operacionalización de las variables y una matriz de concordancia entre los indicadores de la variable "motivación intrínseca" y los ítems que conforman el test de motivación intrínseca.

El cuestionario de estilo de aprendizaje reflexivocrítico, de 78 ítems, se elaboró en base a los aportes de la teoría de estilo de aprendizaje de Honey y Mumford (1986) [12] y la teoría de los estilos de aprendizaje de Kolb (1976) [13]. El propósito del instrumento fue determinar el nivel de desarrollo del estilo de aprendizaje reflexivocrítico en los estudiantes de la Universidad Nacional de Ingeniería.

Se determinó la confiabilidad de los instrumentos de medición (Test de motivación intrínseca y el cuestionario del estilo de aprendizaje reflexivocrítico) a través del método de la división de mitades (la fórmula Spearman - Brown).

Además, la determinación de confiabilidad del test de motivación intrínseca se realizó con un grupo piloto (20 estudiantes) y mediante el método de mitades partidas. El test se dividió en dos mitades comparables (ítems pares e impares) y se calificó por separado cada una de ellas. El coeficiente de correlación obtenido fue de 0.75 .

La confiabilidad del cuestionario del estilo de aprendizaje reflexivo-crítico, en la prueba piloto de 20 estudiantes, por el método de mitades partidas, se utilizó el coeficiente de correlación de Pearson. La confiabilidad del cuestionario resultó 0.82 , lo que significa que el cuestionario tiene un alto índice de confiabilidad (82\%) con un error de medición de 18\%; según las tablas de valores de los niveles de confiabilidad.

\section{RESULTADOS}

Los hallazgos de la investigación responden a la hipótesis Existe una relación significativa entre la motivación intrínseca y el estilo de aprendizaje reflexivo-crítico en los estudiantes de la Universidad Nacional de Ingeniería, mediante la presentación, el análisis e interpretación de los resultados del test de motivación intrínseca y el cuestionario del estilo de aprendizaje reflexivocrítico aplicado a 371 , como se muestra en la Tabla 1 y Tabla 4. Para tal propósito, en un primer momento, se realizó el análisis de datos mediante estadísticos descriptivos con el objetivo de determinar los niveles en que se encuentran los estudiantes en motivación intrínseca y estilo de aprendizaje reflexivo-crítico; luego, en un segundo momento, se realizó el análisis de datos mediante los estadísticos inferenciales con el propósito de establecer correlaciones, con un nivel de significación al 0.05 .

Análisis descriptivo: Este nivel de análisis facilitó el conocimiento de las características del estudio, una vez depurados los datos y analizado las propiedades más relevantes. Con tal fin, se han utilizado las medidas de tendencia central, representativas del conjunto de la distribución y las de variabilidad para saber cómo se agrupan los datos. 
Tabla 1. Resultados sobre el nivel de motivación intrínseca de los estudiantes.

\begin{tabular}{|c|c|c|c|c|c|c|c|c|c|c|c|}
\hline$N^{o}$ & Resultados & $N^{o}$ & Resultados & $N^{o}$ & Resultados & $N^{o}$ & Resultados & $N^{o}$ & Resultados & $N^{o}$ & Resultados \\
\hline 1 & 45.5 & 34 & 60.5 & 67 & 64 & 100 & 59.5 & 133 & 60 & 166 & 66.5 \\
\hline 2 & 43 & 35 & 58 & 68 & 55 & 101 & 64 & 134 & 70 & 167 & 47 \\
\hline 3 & 48.5 & 36 & 46.5 & 69 & 69.5 & 102 & 53 & 135 & 57.5 & 168 & 50 \\
\hline 4 & 68 & 37 & 59.5 & 70 & 51.5 & 103 & 61.5 & 136 & 63.5 & 169 & 56.5 \\
\hline 5 & 44 & 38 & 46.5 & 71 & 49.5 & 104 & 41 & 137 & 43 & 170 & 60 \\
\hline 6 & 54 & 39 & 61 & 72 & 61 & 105 & 71 & 138 & 65 & 171 & 55 \\
\hline 7 & 57.5 & 40 & 51.5 & 73 & 57 & 106 & 53 & 139 & 49.5 & 172 & 52 \\
\hline 8 & 67.5 & 41 & 41.5 & 74 & 53.5 & 107 & 52.5 & 140 & 52.5 & 173 & 66 \\
\hline 9 & 57 & 42 & 36 & 75 & 56 & 108 & 45.5 & 141 & 52.5 & 174 & 47.5 \\
\hline 10 & 66 & 43 & 47 & 76 & 61 & 109 & 58 & 142 & 51 & 175 & 50.5 \\
\hline 11 & 68 & 44 & 30.5 & 77 & 67 & 110 & 56.5 & 143 & 47.5 & 176 & 48 \\
\hline 12 & 56 & 45 & 50 & 78 & 62.5 & 111 & 42.5 & 144 & 47 & 177 & 48.5 \\
\hline 13 & 51.5 & 46 & 67.5 & 79 & 62 & 112 & 67 & 145 & 49 & 178 & 48.5 \\
\hline 14 & 48.5 & 47 & 59.5 & 80 & 45 & 113 & 60 & 146 & 55 & 179 & 65 \\
\hline 15 & 64 & 48 & 70.5 & 81 & 64.5 & 114 & 54.5 & 147 & 44.5 & 180 & 58.5 \\
\hline 16 & 50.5 & 49 & 54.5 & 82 & 38.5 & 115 & 58.5 & 148 & 61 & 181 & 57.5 \\
\hline 17 & 60 & 50 & 61.5 & 83 & 72 & 116 & 63.5 & 149 & 50.5 & 182 & 62 \\
\hline 18 & 51.4 & 51 & 65 & 84 & 52 & 117 & 59 & 150 & 47.5 & 183 & 58.5 \\
\hline 19 & 44.5 & 52 & 62 & 85 & 56.5 & 118 & 59 & 151 & 53.5 & 184 & 59 \\
\hline 20 & 53.5 & 53 & 53 & 86 & 74 & 119 & 61.5 & 152 & 23.5 & 185 & 54.5 \\
\hline 21 & 49 & 54 & 64 & 87 & 42 & 120 & 70.5 & 153 & 57 & 186 & 66 \\
\hline 22 & 40 & 55 & 51.5 & 88 & 64 & 121 & 56.5 & 154 & 61.5 & 187 & 59.5 \\
\hline 23 & 50 & 56 & 38 & 89 & 65 & 122 & 62 & 155 & 62 & 188 & 49.5 \\
\hline 24 & 71.5 & 57 & 60 & 90 & 58 & 123 & 53.5 & 156 & 56.5 & 189 & 59.5 \\
\hline 25 & 63 & 58 & 50 & 91 & 68.5 & 124 & 33 & 157 & 64 & 190 & 58.5 \\
\hline 26 & 33 & 59 & 52 & 92 & 55 & 125 & 47 & 158 & 66.5 & 191 & 61.5 \\
\hline 27 & 35.5 & 60 & 52 & 93 & 47 & 126 & 58.5 & 159 & 60.5 & 192 & 62.5 \\
\hline 28 & 51.5 & 61 & 60.5 & 94 & 76.5 & 127 & 58.5 & 160 & 52 & 193 & 54 \\
\hline
\end{tabular}




\begin{tabular}{ccccccccccccc}
\hline 29 & 46 & 62 & 53 & 95 & 65 & 128 & 58.5 & 161 & 48 & & 194 & 47 \\
30 & 59 & 63 & 45 & 96 & 69 & 129 & 65 & 162 & 47.5 & 195 & 40 & \\
31 & 54 & 64 & 51.5 & 97 & 60.5 & 130 & 51 & 163 & 51 & 196 & 60 \\
32 & 56.5 & 65 & 56.5 & 98 & 60 & 131 & 57 & 164 & 46.5 & 197 & 53.5 \\
33 & 52.5 & 66 & 40.5 & 99 & 63 & 132 & 60 & 165 & 58.5 & 198 & 58.5 \\
\hline
\end{tabular}

\begin{tabular}{|c|c|c|c|c|c|c|c|c|c|c|c|}
\hline$N^{o}$ & Resultados & $N^{o}$ & Resultados & $N^{o}$ & Resultados & $N^{o}$ & Resultados & $N^{o}$ & Resultados & $N^{o}$ & Resultados \\
\hline 199 & 50.5 & 228 & 53 & 257 & 57.5 & 286 & 47.5 & 315 & 43.5 & 344 & 56 \\
\hline 200 & 43.5 & 229 & 62 & 258 & 48.5 & 287 & 51.5 & 316 & 60.5 & 345 & 48.5 \\
\hline 201 & 42.5 & 230 & 48.5 & 259 & 61 & 288 & 63 & 317 & 55 & 346 & 71 \\
\hline 202 & 70 & 231 & 69 & 260 & 64 & 289 & 67.5 & 318 & 58.5 & 347 & 66 \\
\hline 203 & 58.5 & 232 & 63.5 & 261 & 55.5 & 290 & 64 & 319 & 62.5 & 348 & 56 \\
\hline 204 & 51.5 & 233 & 60 & 262 & 52 & 291 & 63 & 320 & 65 & 349 & 60 \\
\hline 205 & 40 & 234 & 65.5 & 263 & 60 & 292 & 60 & 321 & 50 & 350 & 65.5 \\
\hline 206 & 56.5 & 235 & 71.5 & 264 & 69 & 293 & 60 & 322 & 64 & 351 & 55 \\
\hline 207 & 42 & 236 & 62 & 265 & 59 & 294 & 54 & 323 & 60 & 352 & 62 \\
\hline 208 & 51.5 & 237 & 61.5 & 266 & 62 & 295 & 67 & 324 & 54 & 353 & 52 \\
\hline 209 & 40 & 238 & 56.5 & 267 & 68 & 296 & 50.5 & 325 & 69 & 354 & 49 \\
\hline 210 & 66.5 & 239 & 65.5 & 268 & 60 & 297 & 59.5 & 326 & 47 & 355 & 51 \\
\hline 211 & 59.5 & 240 & 42 & 269 & 78 & 298 & 53.5 & 327 & 52 & 356 & 60 \\
\hline 212 & 60.5 & 241 & 68.5 & 270 & 56 & 299 & 61 & 328 & 63.5 & 357 & 71 \\
\hline 213 & 62.5 & 242 & 61.5 & 271 & 56 & 300 & 66.4 & 329 & 59.5 & 358 & 51.5 \\
\hline 214 & 59.5 & 243 & 46 & 272 & 56 & 301 & 45 & 330 & 55 & 359 & 51 \\
\hline 215 & 51.5 & 244 & 55 & 273 & 63.5 & 302 & 43.5 & 331 & 32 & 360 & 54.5 \\
\hline 216 & 61.5 & 245 & 66.5 & 274 & 63.5 & 303 & 55 & 332 & 61.5 & 361 & 58.5 \\
\hline 217 & 60 & 246 & 61 & 275 & 66.5 & 304 & 66 & 333 & 63 & 362 & 61.5 \\
\hline 218 & 51.5 & 247 & 73 & 276 & 60 & 305 & 62 & 334 & 31.5 & 363 & 61 \\
\hline 219 & 52 & 248 & 63.5 & 277 & 61 & 306 & 69 & 335 & 60 & 364 & 61.5 \\
\hline 220 & 55.5 & 249 & 62.5 & 278 & 59.5 & 307 & 62.5 & 336 & 54.5 & 365 & 67.5 \\
\hline 221 & 53 & 250 & 45.5 & 279 & 61 & 308 & 59.5 & 337 & 65.5 & 366 & 54.5 \\
\hline
\end{tabular}




\begin{tabular}{|c|c|c|c|c|c|c|c|c|c|c|c|}
\hline 222 & 61 & 251 & 60.5 & 280 & 65.5 & 309 & 58 & 338 & 47.5 & 367 & 54 \\
\hline 223 & 57.5 & 252 & 65 & 281 & 47 & 310 & 61.5 & 339 & 58 & 368 & 55.5 \\
\hline 224 & 63 & 253 & 57.5 & 282 & 66.5 & 311 & 60 & 340 & 56 & 369 & 68 \\
\hline 225 & 58 & 254 & 61.5 & 283 & 62.5 & 312 & 67 & 341 & 65 & 370 & 53.5 \\
\hline 226 & 57 & 255 & 64.5 & 284 & 62.5 & 313 & 66.5 & 342 & 56.5 & 371 & 55.5 \\
\hline 227 & 66 & 256 & 63 & 285 & 64 & 314 & 61 & 343 & 68.5 & & \\
\hline
\end{tabular}

En la Tabla 2, se puede observar, en forma general, el nivel de motivación intrínseca de los estudiantes de la UNI de una muestra de estudio de 371 (100\%). Observamos que 31 (8.36) corresponde a un nivel muy alto; 183 (49.33\%) presentan un nivel de motivación intrínseca alta;
124 (33.4\%), un nivel medio o moderado; 27 $(7.28 \%)$ un nivel bajo; y 6 (1.6\%), un nivel de motivación muy bajo. El promedio de las puntuaciones en la variable motivación intrínseca que presentan los estudiantes de la UNI es de 58.40, con una desviación estándar de 8.4 puntos.

Tabla 2. Niveles de motivación intrínseca en los estudiantes de ingeniería de la UNI.

\begin{tabular}{|c|c|c|c|c|c|c|}
\hline Nivel & Puntaje & Frecuencia & Porcentaje & $\begin{array}{l}\text { Porcentaje } \\
\text { acumulado }\end{array}$ & Promedio & Desv. Estandar \\
\hline Muy bajo & {$[23.50: 34.40]$} & 6 & $1.62 \%$ & $1.62 \%$ & -- & -- \\
\hline Bajo & {$[34.50: 45.30]$} & 27 & $7.28 \%$ & $8.89 \%$ & -- & -- \\
\hline Promedio - media & {$[45.40: 56.20]$} & 124 & $33.42 \%$ & $42.32 \%$ & -- & -- \\
\hline Alto & {$[56.30: 67.10]$} & 183 & $49.33 \%$ & $91.64 \%$ & -- & -- \\
\hline Muy alto & {$[67.20: 78.00]$} & 31 & $8.36 \%$ & $100.00 \%$ & -- & -- \\
\hline Total & & 371 & $100.00 \%$ & & $\mathbf{5 8 . 4 0}$ & 8.42393348 \\
\hline
\end{tabular}


Fig.1 Nivel de motivación intrínseca en los estudiantes de la UNI.

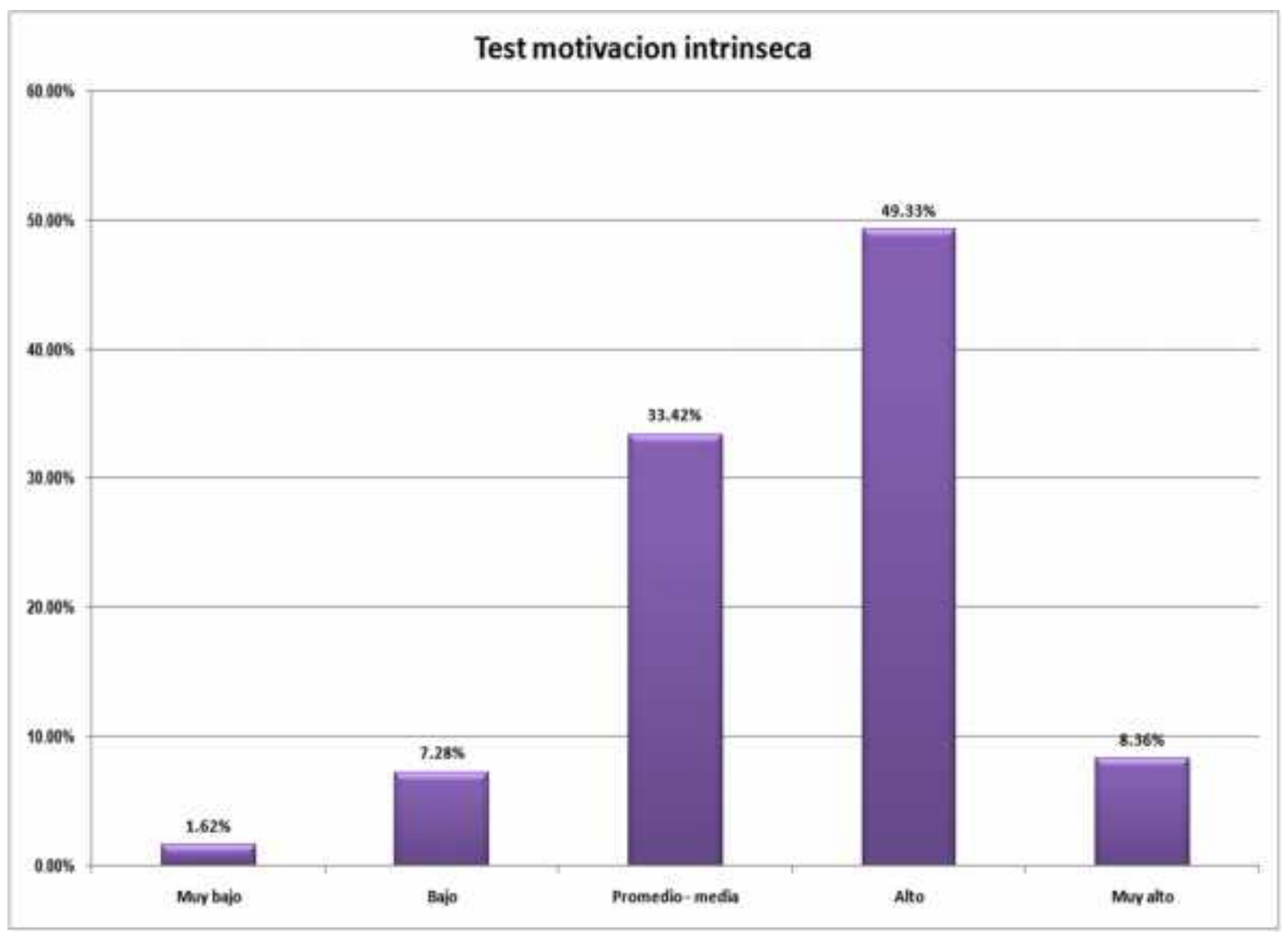

En la Figura 1 se observa los niveles de motivación intrínseca de los estudiantes de la UNI. De 371 estudiantes evaluados, 31 estudiantes (8.36 $\%)$ se encuentran en un nivel muy alto; 183 (49\%), en un nivel alto; 124 (33.4\%), en un nivel medio (promedio); 27 (7.28\%), en un nivel bajo; y 6 estudiantes $(1.6 \%)$, en un nivel muy bajo. Esto indica que un mayor porcentaje de estudiantes de ingeniería alcanzan un nivel alto y medio en motivación intrínseca y un menor porcentaje alcanzan un nivel bajo y muy bajo.
El nivel de motivación intrínseca exhibido por los estudiantes de la UNI tiende a ser elevado como lo indican los resultados del test en la Tabla 3.

El 50\% de los estudiantes está por encima del valor 58.50 y el restante $50 \%$, por debajo de este valor. El puntaje más frecuente de la muestra es de 60 puntos, el puntaje máximo, de 78.0 puntos y un mínimo, de 23.50. El rango para esta investigación varió de 34.30 a 78.00. Asimismo, se desvían de 58.40 , en promedio, a 8.4 puntos. 
Tabla 3. Resultado global del test de motivación intrínseca.

\begin{tabular}{ll}
\multicolumn{1}{c}{ Estadígrafos } & \multicolumn{1}{c}{ MI } \\
\hline Media & 58.40 \\
Mediana & 58.50 \\
Moda & 60.00 \\
Puntaje Max. & 78.00 \\
Puntaje Min. & 23.50 \\
Rango & $34.30-78.00$ \\
Varianza & 70.96265521 \\
Desv. Estándar & 8.423933476 \\
Kurtosis & 0.624771043 \\
Asimetría & 0.55752 \\
\hline
\end{tabular}

Ver Tabla 4, los resultados sobre el nivel de crítico de los estudiantes de ingeniería de la UNI. desarrollo del estilo de aprendizaje reflexivo-

Tabla 4 Los resultados del cuestionario estilo del aprendizaje reflexivo-crítico (EARC).

\begin{tabular}{llllllllllll}
\hline$N^{\boldsymbol{o}}$ & Resultados & $\boldsymbol{N}^{\boldsymbol{o}}$ & Resultados & $\boldsymbol{N}^{\boldsymbol{o}}$ & Resultados & $\boldsymbol{N}^{\boldsymbol{o}}$ & Resultados & $\boldsymbol{N}^{\boldsymbol{o}}$ & Resultados & $\boldsymbol{N}^{\boldsymbol{o}}$ & Resultados $^{2}$ \\
\hline 1 & 48 & 34 & 122 & 67 & 154.5 & 100 & 169 & 133 & 177 & 166 & 140.5 \\
2 & 125 & 35 & 138 & 68 & 172 & 101 & 109 & 134 & 146.5 & 167 & 151.5 \\
3 & 115 & 36 & 114 & 69 & 134.5 & 102 & 142.5 & 135 & 132.5 & 168 & 118 \\
4 & 115.5 & 37 & 123 & 70 & 162.5 & 103 & 193 & 136 & 147 & 169 & 148.5 \\
5 & 125.5 & 38 & 170.5 & 71 & 82 & 104 & 158.5 & 137 & 168.5 & 170 & 110 \\
6 & 141.5 & 39 & 161 & 72 & 137 & 105 & 163.5 & 138 & 123 & 171 & 95.5 \\
7 & 64 & 40 & 101 & 73 & 115 & 106 & 117.5 & 139 & 144.5 & 172 & 146 \\
8 & 136 & 41 & 61 & 74 & 139 & 107 & 128 & 140 & 138 & 173 & 168 \\
9 & 145.5 & 42 & 56 & 75 & 125 & 108 & 149 & 141 & 112 & 174 & 134 \\
10 & 131.5 & 43 & 116 & 76 & 105.5 & 109 & 190 & 142 & 122.5 & 175 & 129.5 \\
11 & 162 & 44 & 49 & 77 & 144.5 & 110 & 126 & 143 & 115 & 176 & 123 \\
\hline
\end{tabular}




\begin{tabular}{|c|c|c|c|c|c|c|c|c|c|c|c|}
\hline 12 & 151.5 & 45 & 118.5 & 78 & 141 & 111 & 146 & 144 & 125 & 177 & 117 \\
\hline 13 & 136 & 46 & 98 & 79 & 111.5 & 112 & 153 & 145 & 111 & 178 & 120.5 \\
\hline 14 & 137.5 & 47 & 143.5 & 80 & 137.5 & 113 & 133.5 & 146 & 98.5 & 179 & 162.5 \\
\hline 15 & 63.5 & 48 & 155.5 & 81 & 148 & 114 & 145 & 147 & 152 & 180 & 119 \\
\hline 16 & 114.5 & 49 & 106 & 82 & 52.5 & 115 & 150 & 148 & 130 & 181 & 125.5 \\
\hline 17 & 187.5 & 50 & 158 & 83 & 168 & 116 & 99 & 149 & 115 & 182 & 214 \\
\hline 18 & 77.5 & 51 & 121 & 84 & 156.5 & 117 & 201.5 & 150 & 129.5 & 183 & 151 \\
\hline 19 & 76.5 & 52 & 137 & 85 & 48.5 & 118 & 149 & 151 & 151.5 & 184 & 126 \\
\hline 20 & 140 & 53 & 123 & 86 & 127 & 119 & 127 & 152 & 108 & 185 & 152 \\
\hline 21 & 102.5 & 54 & 176.5 & 87 & 113.5 & 120 & 115.5 & 153 & 115.5 & 186 & 152 \\
\hline 22 & 120.5 & 55 & 40.5 & 88 & 138.5 & 121 & 121.5 & 154 & 47 & 187 & 151 \\
\hline 23 & 136.5 & 56 & 65.5 & 89 & 128 & 122 & 134.5 & 155 & 149 & 188 & 159 \\
\hline 24 & 155.5 & 57 & 116 & 90 & 123.5 & 123 & 61.5 & 156 & 154 & 189 & 185.5 \\
\hline 25 & 111 & 58 & 119 & 91 & 129.5 & 124 & 81.5 & 157 & 164 & 190 & 118.5 \\
\hline 26 & 46 & 59 & 145 & 92 & 137 & 125 & 142 & 158 & 113 & 191 & 193 \\
\hline 27 & 104.5 & 60 & 131 & 93 & 180.5 & 126 & 150 & 159 & 168.5 & 192 & 133 \\
\hline 28 & 13 & 61 & 118 & 94 & 138 & 127 & 108.5 & 160 & 105.5 & 193 & 150 \\
\hline 29 & 95.5 & 62 & 131 & 95 & 132 & 128 & 162 & 161 & 162 & 194 & 157 \\
\hline 30 & 141 & 63 & 98.5 & 96 & 100.5 & 129 & 157.5 & 162 & 145.5 & 195 & 141.5 \\
\hline 31 & 148 & 64 & 149 & 97 & 111.5 & 130 & 145.5 & 163 & 121.5 & 196 & 111 \\
\hline 32 & 168 & 65 & 149.5 & 98 & 143.5 & 131 & 129.5 & 164 & 125 & 197 & 94 \\
\hline 33 & 146 & 66 & 118 & 99 & 146.5 & 132 & 161 & 165 & 125 & 198 & 113.5 \\
\hline
\end{tabular}

\begin{tabular}{|c|c|c|c|c|c|c|c|c|c|c|c|}
\hline$N^{o}$ & Resultados & $N^{o}$ & Resultados & $N^{o}$ & Resultados & $N^{o}$ & Resultados & $N^{o}$ & Resultados & $N^{o}$ & Resultados \\
\hline 199 & 130.5 & 228 & 80 & 257 & 105.5 & 286 & 130.5 & 315 & 128 & 344 & 105.5 \\
\hline 200 & 149.5 & 229 & 124.5 & 258 & 133.5 & 287 & 107.5 & 316 & 69 & 345 & 174 \\
\hline 201 & 144 & 230 & 116 & 259 & 160.5 & 288 & 175 & 317 & 94 & 346 & 100.5 \\
\hline 202 & 144 & 231 & 178 & 260 & 118 & 289 & 109.5 & 318 & 84 & 347 & 87.5 \\
\hline 203 & 101 & 232 & 167.5 & 261 & 133.5 & 290 & 132 & 319 & 161.5 & 348 & 151.5 \\
\hline 204 & 87 & 233 & 146.5 & 262 & 126 & 291 & 158 & 320 & 123.5 & 349 & 124.5 \\
\hline
\end{tabular}




\begin{tabular}{|c|c|c|c|c|c|c|c|c|c|c|c|}
\hline 205 & 80 & 234 & 111 & 263 & 128 & 292 & 130.5 & 321 & 130 & 350 & 122.5 \\
\hline 206 & 163.5 & 235 & 132.5 & 264 & 148 & 293 & 113.5 & 322 & 154.5 & 351 & 122.5 \\
\hline 207 & 144.5 & 236 & 109.5 & 265 & 111 & 294 & 128.5 & 323 & 123 & 352 & 139 \\
\hline 208 & 128.5 & 237 & 133 & 266 & 133 & 295 & 177.5 & 324 & 144 & 353 & 122 \\
\hline 209 & 83.5 & 238 & 123 & 267 & 178 & 296 & 145 & 325 & 126.5 & 354 & 92.5 \\
\hline 210 & 106.5 & 239 & 155 & 268 & 134 & 297 & 137 & 326 & 135.5 & 355 & 93.5 \\
\hline 211 & 89 & 240 & 172.5 & 269 & 145.5 & 298 & 139 & 327 & 141 & 356 & 48.5 \\
\hline 212 & 88.5 & 241 & 129.5 & 270 & 164 & 299 & 99.5 & 328 & 125.5 & 357 & 153.5 \\
\hline 213 & 106 & 242 & 146.5 & 271 & 158 & 300 & 163 & 329 & 145 & 358 & 118.5 \\
\hline 214 & 118.5 & 243 & 122.5 & 272 & 126.5 & 301 & 100 & 330 & 159.5 & 359 & 179.5 \\
\hline 215 & 147.5 & 244 & 152 & 273 & 144 & 302 & 141 & 331 & 153.5 & 360 & 128 \\
\hline 216 & 137 & 245 & 83.5 & 274 & 165.5 & 303 & 137 & 332 & 109 & 361 & 169.5 \\
\hline 217 & 131.5 & 246 & 173 & 275 & 113 & 304 & 137.5 & 333 & 161.5 & 362 & 185 \\
\hline 218 & 190 & 247 & 135.5 & 276 & 186 & 305 & 182.5 & 334 & 126 & 363 & 40.5 \\
\hline 219 & 103 & 248 & 94.5 & 277 & 119 & 306 & 149 & 335 & 153.5 & 364 & 166.5 \\
\hline 220 & 164 & 249 & 142 & 278 & 140 & 307 & 149.5 & 336 & 109 & 365 & 112 \\
\hline 221 & 157 & 250 & 157 & 279 & 140.5 & 308 & 156 & 337 & 161.5 & 366 & 156 \\
\hline 222 & 143 & 251 & 168.5 & 280 & 137 & 309 & 135 & 338 & 126 & 367 & 163.5 \\
\hline 223 & 150.5 & 252 & 189.5 & 281 & 105.5 & 310 & 105 & 339 & 153.5 & 368 & 137.5 \\
\hline 224 & 138 & 253 & 68 & 282 & 137 & 311 & 141.5 & 340 & 143 & 369 & 112 \\
\hline 225 & 124 & 254 & 159 & 283 & 179.5 & 312 & 98.5 & 341 & 141 & 370 & 113.5 \\
\hline 226 & 140.5 & 255 & 130 & 284 & 135.5 & 313 & 161 & 342 & 175 & 371 & 121 \\
\hline 227 & 81.5 & 256 & 121.5 & 285 & 140.5 & 314 & 104 & 343 & 176.5 & & \\
\hline
\end{tabular}

En la Tabla 5, se puede observar en cuanto al nivel de desarrollo del estilo de aprendizaje reflexivocrítico de los estudiantes de la UNI, de 371 $(100 \%), 24$ estudiantes $(6.5 \%)$ presenta un nivel muy alto de desarrollo del estilo de aprendizaje reflexivo-crítico; 161 (43.4\%), un nivel alto; 153 (41.2\%), un nivel medio o moderado; 23 (6.2\%), un nivel bajo; y 10 (2.7\%), un nivel muy bajo. 
Tabla 5. Nivel de desarrollo del estilo de aprendizaje reflexivo - critico en los estudiantes de la UNI.

\begin{tabular}{llllll}
\hline \multicolumn{1}{c}{ Nivel } & \multicolumn{1}{c}{ Puntajes } & Frecuencia & Porcentaje & Promedio & Desv. Estandar \\
\hline Muy alto & {$[173.80: 214.00]$} & 24 & $6.5 \%$ & \\
Alto & {$[133.60: 173.70]$} & 161 & $43.4 \%$ & \\
Moderado & {$[93.40: 133.50]$} & 153 & $41.2 \%$ & \\
\hline Bajo & {$[53.20: 93.30]$} & 23 & $6.2 \%$ & \\
Muy bajo & {$[13.00: 53.10]$} & 10 & $2.7 \%$ & & \\
Total & & $\mathbf{3 7 1}$ & $\mathbf{1 0 0 . 0 \%}$ & $\mathbf{1 3 3 . 7 0}$ & $\mathbf{3 0 . 2 3 9 0 1 2 1 9}$ \\
\hline
\end{tabular}

En la Fig. 2, se observa los niveles de desarrollo del estilo de aprendizaje reflexivo-crítico de los estudiantes de la UNI, donde de 371 estudiantes evaluados, 24 estudiantes, que representa un 6.5 $\%$, se encuentran en un nivel muy alto; 161 estudiantes $(43.4 \%)$, en un nivel alto; 153 (41.2 $\%)$, en un nivel medio; 23 estudiantes $(6.2 \%)$, en un nivel bajo; y 10 estudiantes $(2.7 \%)$, en un nivel muy bajo. Esto indica que un mayor porcentaje de estudiantes alcanzan un nivel alto y moderado en el desarrollo del estilo de aprendizaje reflexivocrítico y en un menor porcentaje alcanzan un nivel bajo y muy bajo.

Fig. 2 Nivel de desarrollo del estilo de aprendizaje reflexivo - crítico en los estudiantes de la UNI.

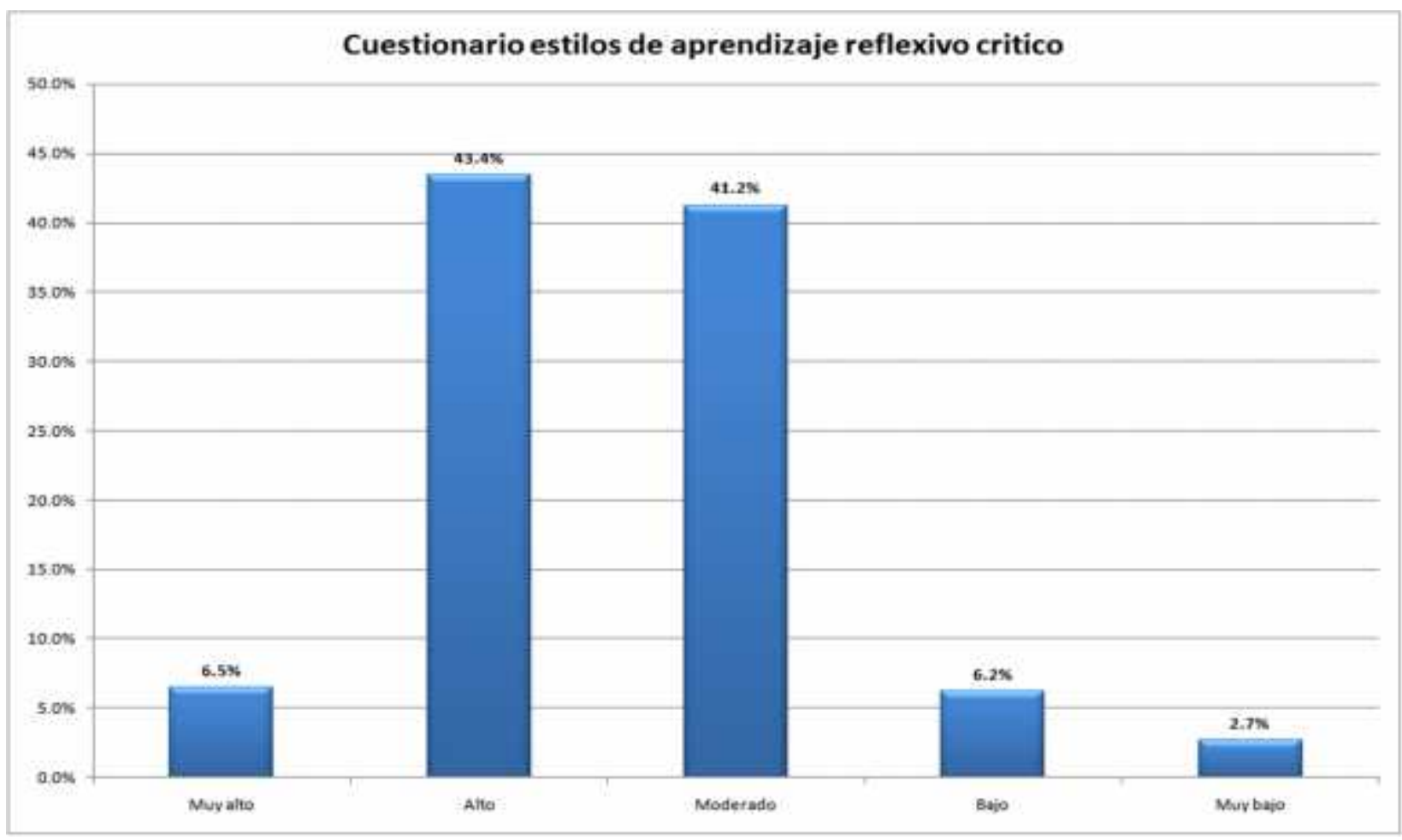


El nivel de desarrollo del estilo de aprendizaje reflexivo-crítico exhibido por los estudiantes de la UNI, tiende a ser elevado como indican los resultados del cuestionario del estilo de aprendizaje reflexivo-crítico, ver Tabla 6. El 50\% de los estudiantes está por encima del valor 133.50 y el restante $50 \%$ se sitúa por debajo de este valor. Los estudiantes de la UNI presentan un puntaje promedio en el cuestionario de estilo de aprendizaje reflexivo-crítico (EARC) de 133.70. El puntaje más frecuente es de 137. El puntaje máximo del cuestionario de estilo de aprendizaje reflexivo-crítico es de 214 puntos y el mínimo, de 13 puntos. El rango para esta investigación varía de 53.20 a 214. Asimismo se dispersa en promedio, respecto a la media, en 30.2 puntos.

Tabla 6. Resultado global del cuestionario del estilo del aprendizaje reflexivo crítico.

\begin{tabular}{ll}
\multicolumn{1}{c}{ Estadígrafos } & \multicolumn{1}{c}{ EARC } \\
\hline Media & 133.70 \\
Mediana & 133.50 \\
Moda & 137.00 \\
Puntaje Max. & 214.00 \\
Puntaje Min. & 13.00 \\
Rango & $53.20-214.0$ \\
Varianza & 914.3978582 \\
Desv. Estándar & 30.23901219 \\
Kurtosis & 1.067801268 \\
Asimetría & 0.334543 \\
\hline
\end{tabular}

Análisis inferencial.- Para el análisis inferencial con el propósito de contrastar la hipótesis general se empleó el estadístico paramétrico de la prueba de momento de $\mathrm{r}$ de Pearson, Tabla 7. Asimismo para contrastar la hipótesis específica se empleó estadístico no paramétrico como la prueba de Chi
Cuadrada de independencia y el análisis de correlación de rango de Spearman (Rho de Sperman), los resultados de la prueba se presentan en tablas y gráficos a fin de facilitar su comprensión. 
Tabla 7 Coeficiente de correlación producto - momento de Pearson entre la motivación intrínseca y el estilo del aprendizaje reflexivo - crítico en estudiantes de la UNI.

\begin{tabular}{|c|c|c|c|c|}
\hline \multicolumn{5}{|c|}{ Producto } \\
\hline & & & $\begin{array}{l}\text { Calificación } \\
\text { motivación } \\
\text { intrínseca }(X)\end{array}$ & $\begin{array}{l}\text { Calificación_ estilo de } \\
\text { aprendizaje reflexivo } \\
\text { crítico }(Y)\end{array}$ \\
\hline \multirow{5}{*}{\multicolumn{2}{|c|}{$\begin{array}{l}\text { Calificación } \\
\text { motivación } \\
\text { intrínseca }\end{array}$}} & Correlación de Pearson & 1 & $.134(* *)$ \\
\hline & & Sig. (bilateral) & & .010 \\
\hline & & $\begin{array}{l}\text { Suma de cuadrados y productos } \\
\text { cruzados }\end{array}$ & 26256.182 & 29022.201 \\
\hline & & Covarianza & 70.963 & 78.438 \\
\hline & & $\mathrm{N}$ & 371 & 371 \\
\hline \multirow{5}{*}{\multicolumn{2}{|c|}{$\begin{array}{l}\text { Calificación estilo } \\
\text { de aprendizaje } \\
\text { reflexivo critico }\end{array}$}} & Correlación de Pearson & $.134(* *)$ & 1 \\
\hline & & Sig. (bilateral) & .010 & \\
\hline & & $\begin{array}{l}\text { Suma de cuadrados y productos } \\
\text { cruzados }\end{array}$ & 29022.201 & 1784430.712 \\
\hline & & Covarianza & 78.438 & 4822.786 \\
\hline & & $\mathrm{N}$ & 371 & 371 \\
\hline
\end{tabular}

** La correlación es significativa al nivel 0,05 (bilateral).

Resultados generales acerca de la relación entre la motivación intrínseca $y$ el estilo de aprendizaje reflexivo-crítico en estudiantes de la UNI.

Los resultados que se observan en la Tabla 7 , muestra que el valor de $\mathrm{r}$ calculado (0.134) es positivo; entonces la relación entre la motivación intrínseca y el estilo de aprendizaje reflexivocrítico en los estudiantes de la UNI es directa.

También, el valor de $\mathrm{r}(0.134)$ nos indica que la correlación entre la clasificación de la motivación intrínseca y la calificación del estilo de aprendizaje reflexivo-crítico es significativa, porque la significancia (0.010) es menor del valor 0.05 (95\%) de confianza de que la correlación sea verdadera y $5 \%$ de probabilidad de error.

Resultados específicos acerca de la relación entre el nivel de motivación intrínseca y el nivel de desarrollo del estilo de aprendizaje reflexivo-crítico en estudiantes de la UNI

Tabla 8. Coeficiente de correlación Rho de Spearman entre el nivel de motivación intrínseca y el nivel de desarrollo del estilo del aprendizaje reflexivo - crítico. 


\begin{tabular}{|c|c|c|c|c|}
\hline & & & $\begin{array}{l}\text { Calificación } \\
\text { motivación } \\
\text { intrínseca }\end{array}$ & $\begin{array}{l}\text { Calificación estilo de } \\
\text { aprendizaje reflexivo crítico }\end{array}$ \\
\hline \multirow{6}{*}{$\begin{array}{l}\text { Rho de } \\
\text { Spearm } \\
\text { an }\end{array}$} & \multirow{3}{*}{$\begin{array}{l}\text { Calificación } \\
\text { motivación } \\
\text { intrínseca }\end{array}$} & $\begin{array}{l}\text { Coeficiente } \quad \mathrm{de} \\
\text { correlación }\end{array}$ & 1.000 & $.185(* *)$ \\
\hline & & Sig. (bilateral) & . & .000 \\
\hline & & $\mathrm{N}$ & 371 & 371 \\
\hline & \multirow{3}{*}{$\begin{array}{l}\text { Calificación } \\
\text { estilo de } \\
\text { aprendizaje } \\
\text { reflexivo crítico }\end{array}$} & $\begin{array}{l}\text { Coeficiente } \quad \mathrm{de} \\
\text { correlación }\end{array}$ & $.185(* *)$ & 1.000 \\
\hline & & Sig. (bilateral) & .000 & . \\
\hline & & $\mathrm{N}$ & 371 & 371 \\
\hline
\end{tabular}

En la Tabla 8, se puede observar un coeficiente de correlación de Spearman de 0.185 , lo cual indica que la correlación entre el nivel de motivación y el nivel de desarrollo del estilo de aprendizaje reflexivo-crítico es positiva y significativa. El valor de $\mathrm{P}=0.000$, menor a un $\alpha=0.05$. Como el nivel crítico es menor que el nivel de significación establecido se rechaza la hipótesis nula de independencia y se concluye que existe una relación lineal entre las variables.

Tabla 9. Resultados de la prueba de Chi cuadrada de independencia.

\begin{tabular}{|c|c|c|c|}
\hline & Valor & $\mathrm{gl}$ & $\begin{array}{ll}\text { Sig. } & \text { asintótica } \\
\text { (bilateral) } & \end{array}$ \\
\hline Chi-cuadrado de Pearson & 44.091(a) & 16 & .000 \\
\hline Razón de verosimilitudes & 21.262 & 16 & .169 \\
\hline $\begin{array}{l}\text { Asociación lineal por } \\
\text { lineal }\end{array}$ & 9.071 & 1 & .003 \\
\hline $\mathrm{N}$ de casos válidos & 371 & & \\
\hline
\end{tabular}

a 20 casillas $(80.0 \%)$ tienen una frecuencia esperada inferior a 5. La frecuencia mínima esperada es .02.
En la Tabla 9, se observa que el valor obtenido de chi-cuadrada para $x_{o b t}^{2}(44.09)$ es mayor que el 
valor teórico o tabulado para $x_{\text {critico }}^{2}$ (26.9) (0.000 $<0.05$ ) por lo cual se rechaza la hipótesis nula; es decir, se acepta la hipótesis alterna. Por lo tanto, existe una relación significativa entre el nivel de motivación intrínseca y el nivel de desarrollo del estilo de aprendizaje reflexivo-crítico de los estudiantes de ingeniería de la Universidad Nacional de Ingeniería.

\section{RESULTADOS}

Los resultados obtenidos en cada una de las pruebas estadísticas realizadas representan una información relevante que demuestran la existencia de una relación significativa entre la motivación intrínseca y el estilo de aprendizaje reflexivo-crítico en los estudiantes de la Universidad Nacional de Ingeniería.

En el análisis estadístico hecho para cada una de las variables estudiadas se observa lo siguiente:

En el primer objetivo específico, los resultados del test de motivación intrínseca, como se ve en la Tabla 2 y la Figura 1, permiten apreciar que el nivel de motivación intrínseca de los estudiantes de la UNI, presentan el siguiente cuadro: 31 estudiantes $(8.36 \%)$ se encuentran en un nivel muy alto; 183 (49\%), en un nivel alto; 124 estudiantes (33.4\%), en un nivel medio; 27 estudiantes (7.28\%), en un nivel bajo; y 6 estudiantes (1.6\%), en un nivel muy bajo. El promedio que presentan los estudiantes de la UNI es de 58.4 y con una desviación estándar de 8.42 puntos.

Este resultado tiene sustento en las teorías de la evaluación cognitiva, teoría de la autodeterminación y teoría de la automotivación.

Motivar al estudiante es orientarlo en una dirección y asegurar que siga los pasos necesarios para alcanzar el o los objetivos. Motivarse implica la búsqueda de la satisfacción por voluntad propia o a través de la estimulación para accionar intencionalmente y lograr la meta.

Los estudiantes orientados hacia objetivos o metas de aprendizaje tienen la intención, al momento de aprender, de obtener conocimientos, de desarrollar su competencia. Están convencidos que los problemas difíciles no se constituyen en obstáculos sino, más bien, se convierten en un reto o desafío a enfrentar, pensando en el error de manera constructiva e implicándose más en estrategias de pensamiento reflexivo y pensamiento crítico.

Coincidiendo con afirmaciones de Selfert (en Valle Arias, 1997) [14] pensamos que los estudiantes orientados intrínsecamente se comportan de manera autorregulada. Se hallan potenciados a un mayor uso de estrategias; confían en sí mismos; se implican y comprometen en problemas que conlleven desafíos, atribuyéndose a sí mismos el mérito del éxito. La motivación genera un alto compromiso cognitivo, emocional o conductual con la tarea y éste es el que impulsa el acto de aprender significativamente. Por consiguiente, la motivación intrínseca posee una orientación interna que implica la autodeterminación, la autonomía, los sentimientos de competencia, el reto óptimo de la actividad, la curiosidad y la satisfacción.

Tanto Csikszentmihalyi (1993) [15] como Ryan et al (1995) coinciden en señalar que la motivación intrínseca es un fenómeno particular que refleja el potencial positivo de la naturaleza humana. $\mathrm{La}$ motivación intrínseca es una tendencia inherente a buscar novedad y el desafío, a extender y ejercitar las propias capacidades, a explorar, a aprender.

Los estudios de campo han mostrado que los docentes que apoyan la autonomía catalizan una mayor motivación intrínseca, curiosidad y el deseo de desafío en sus estudiantes (Barrett, 1990; Ryan Groinick, 1986) [16]. La teoría de la evaluación cognitiva sugiere que los ambientes sociales pueden facilitar o entorpecer la motivación intrínseca al apoyar o frustrar las necesidades psicológicas innatas de las personas. Los fuertes vínculos entre la motivación intrínseca y la satisfacción de las necesidades de autonomía y competencia han sido demostrados claramente por 
una serie de estudios. Las personas estarán motivadas intrínsecamente sólo por las tareas por las que mantienen un interés intrínseco, actividades que tienen el atractivo de la novedad, el desafío o valor estético.

Por lo expuesto, se puede concluir que 183 (49\%) estudiantes de la UNI presentan un nivel alto de motivación intrínseca; seguido de 124 estudiantes (33.4\%), un nivel medio; mientras que 31estudiantes $(8.36 \%)$, un nivel muy alto; 27 estudiantes (7.28\%), un nivel bajo; y por último el $1.6 \%$, un nivel muy bajo. Resulta vital incrementar la motivación intrínseca media, asimismo fortalecer y mantener el nivel alto y muy alto de motivación intrínseca, porque promueve el desarrollo efectivo del estilo de aprendizaje reflexivo-crítico y el aprendizaje significativo. Esto contribuye al mejoramiento de la calidad formativa científico-tecnológica y la calidad profesional de los estudiantes de la UNI.

Teniendo en cuenta los resultados del cuestionario del estilo de aprendizaje reflexivo-crítico, se puede señalar con respecto al segundo objetivo específico, como se observa en la tabla 5 y la figura 2, que un porcentaje de $43.4 \%$ de estudiantes de la UNI se ubican en niveles altos, el $41.2 \%$, en niveles medios; y un porcentaje minoritario $(6.5 \%, 6.2 \%$ y $2.7 \%)$ de estudiantes, en niveles muy alto, bajo y muy bajo. Se encontró que el promedio de las puntuaciones en la variable estilos de aprendizaje reflexivo-crítico de los estudiantes de la UNI es de 133.70 y con una desviación estándar de 30.23.

La literatura especializada sobre la variable estilos de aprendizaje reflexivo-crítico en estudiantes universitarios introduce diversas perspectivas sobre la necesidad de formar estudiantes capaces de reflexionar y cuestionar críticamente sobre su ser y saber hacer en el ejercicio de la profesión de ingenieros, se aspira a la interpretación de la realidad en los ámbitos donde desarrollan sus prácticas. Los futuros ingenieros deben desarrollar competencias para desempeñarse como profesionales reflexivos y críticos de su práctica.
Se trata de integrar pensamiento y acción. Los estudiantes deben asumir la reflexión y la criticidad en, desde y sobre la práctica.

Para Dewey (1989) [17], el pensamiento reflexivo y la eficacia del estilo de aprendizaje reflexivo son logros del individuo. Para conseguir hay que poner en duda o cuestionar nuestras ideas preconcebidas. Asimismo señala que la tarea del sujeto reflexivo consiste en tener claridad sobre los prejuicios y dar algún orden a la cadena de pensamiento que nos acaece cuando pensamos. La reflexión o el estilo de aprendizaje reflexivo son la liberación de la rutina, la búsqueda de acciones inteligentes y el enriquecimiento de significados en los individuos. La función del pensamiento reflexivo desmenuza los procesos internos que produce la reflexión: inferencias, comprobación, búsqueda de evidencias, realización de acciones para comprobar que el pensamiento ha tenido lugar.

La reflexión como proceso de pensamiento deliberado, racional y crítico se traduce en el examen, la argumentación, la deliberación y toma de decisiones respecto a nuestras actuaciones. Se asume como un proceso de revisión; reflexionar implica un acto de pensamiento. Es abstraerse para observar, debatir consigo mismo y tratar de explicar las propias acciones, es mirar críticamente lo que se hace, justificar cada una de las decisiones tomadas y profundizar desde el cuestionamiento propio a fin de rectificar a tomar decisiones que pretenden en todo caso, mejorar la praxis académica.

Según Alonso (1994) [18], el estilo de aprendizaje reflexivo constituye el estilo de razonamiento donde predomina la observación y el análisis de los resultados de la experiencia realizada. Los sujetos reflexivos gustan considerar las experiencias y observarlas desde distintas perspectivas. Reúnen datos, analizándolos con detenimiento antes de llegar a alguna conclusión. Los alumnos que obtengan un predominio claro del estilo reflexivo se caracterizan por ser ponderados, prudentes, detallistas, elaboradores de argumentos, estudiosos de comportamientos, 
distantes, inquisidores, investigadores y previsores de alternativas, etc.

Para Kurland (1995) [19], el pensar críticamente y el estilo de aprendizaje crítico están relacionado con la razón, la honestidad intelectual y la amplitud mental del sujeto. Pensar críticamente involucra seguir el hilo de las evidencias hasta donde ellas nos lleven, tener en cuenta todas las posibilidades, confiar en la razón, considerar alguna gama de posibles puntos de vista $y$ explicaciones, sopesar los efectos de las posibles motivaciones y prejuicios, estar más interesados en encontrar la verdad que en tener la razón, no rechazar ningún punto de vista, estar conscientes de nuestros sesgos y prejuicios para que influyan en nuestro juicio.

Los alumnos que consigan un predominio del estilo de aprendizaje crítico se caracterizan por presentar el siguiente perfil: analítico crítico, razonador dialéctico, teórico divergente, juzgador, autorregulador, discernidor y clarificador, evaluador de argumentos, buscador de razones adecuadas, sintético, comparador, argumentador, explorador reflexivo, planificador y supervisor de su estudio, tomador de decisiones efectivas, elaborador de inferencias, elaborador y formulador de preguntas evaluativas, comprobador, deductor de conclusiones, investigador crítico. Asimismo, el sujeto crítico posee perseverancia intelectual, independencia intelectual, flexibilidad mental, cuestionador, tolerante. Tiene curiosidad, actitud abierta, muestra sistematicidad y objetividad, coraje intelectual, inquisitivo, ordenado y concienzudo, muestra una actitud de suspender juicios y sentido de justicia.

Por lo tanto, se puede concluir que el $43.4 \%$ de los estudiantes de ingeniería presentan un nivel alto de desarrollo del estilo de aprendizaje reflexivocrítico; un $41.2 \%$, un nivel medio; habiendo diferencias significativas entre el nivel muy alto y el nivel bajo y muy bajo. Resulta, entonces, importante incrementar el nivel de estilo de aprendizaje reflexivo-crítico medio; asimismo, fortalecer y mantener el nivel alto y muy alto.
El objetivo del trabajo era determinar el grado de relación existente entre la motivación intrínseca y el estilo de aprendizaje reflexivo-crítico en los estudiantes de la Universidad Nacional de Ingeniería y la hipótesis central que planteamos fue la necesidad de una relación significativa entre la motivación intrínseca y el estilo de aprendizaje reflexivo-crítico en los estudiantes de ingeniería de la UNI.

Teniendo en cuenta los resultados, se puede señalar, con respecto a la hipótesis central $(\mathrm{H}$. principal), tal como se ve en la tabla 7 , que existe una correlación positiva y directa, debido a que el coeficiente de correlación producto-momento de Pearson es de 0.134. Estos hallazgos significan que las variaciones en una de las variables irán acompañadas de variaciones en la otra variable. Asimismo, se confirma la existencia de una relación significativa entre la motivación intrínseca y el estilo de aprendizaje reflexivo-crítico.

El análisis estadístico realizado en relación con la hipótesis central nos permite rechazar la Ho y aceptar la hipótesis alternativa al confirmarse que el valor de $\mathrm{r}(0.134)$ nos indica que la correlación entre la motivación intrínseca y estilo de aprendizaje reflexivo-crítico es significativa porque la significancia $(0.010)$ es menor del valor 0.05 (95\% de confianza de que la correlación es verdadera y $5 \%$ de probabilidad de error). Por tanto, concluimos que existe una relación significativa entre la motivación intrínseca y el estilo de aprendizaje reflexivo-crítico en los estudiantes de la UNI.

Los resultados que arroja la prueba de coeficiente de correlación de Rho de Spearman nos indica, con respecto a la hipótesis específica 1: "Existe una relación significativa entre el nivel de motivación intrínseca y el nivel de desarrollo del estilo del aprendizaje reflexivo-crítico en los estudiantes de ingeniería de la Universidad Nacional de Ingeniería", como se ve en la tabla 8 , que la correlación entre el nivel de motivación intrínseca y el nivel de desarrollo del estilo de aprendizaje reflexivo-crítico, es positiva y significativa, porque 
el coeficiente de correlación es de 0.185. Este resultado se fundamenta en la teoría de la motivación intrínseca y la teoría del estilo de aprendizaje reflexivo-crítico. Asimismo, este resultado se condice con algunos estudios científicos (Belenky y otros, 1986; Dewey, 1985: Deci y Ryan, 1995) [20].

Teniendo en cuenta los resultados de la prueba de Chi-cuadrado, se puede señalar, con respecto a la misma hipótesis específica 1 , como se muestra en la tabla 9, que existe una relación significativa entre el nivel de motivación intrínseca y el nivel de desarrollo de estilo de aprendizaje reflexivo-crítico en los estudiantes de la UNI.

El análisis estadístico efectuado nos permite rechazar la Ho y aceptar la hipótesis alternativa, porque el valor obtenido de chi-cuadrada $x_{o b t}^{2}$ (44.09) es mayor que el valor teórico o tabulado $\mathrm{X}^{2}$ crítico $=26.9(0.000<0.05)$. Por lo tanto se concluye una relación significativa entre el nivel de motivación intrínseca y el nivel de desarrollo del estilo de aprendizaje reflexivo-crítico de los estudiantes de la UNI.

El resultado de nuestra investigación es similar a los resultados obtenidos por otros investigadores tales como: Roces Montero, Gonzales y Álvarez, 2000; y Palomino, 2009 et al.

Roces Montero, Gonzales y Álvarez et al (2000) investigaron las relaciones entre la motivación, estrategias de aprendizaje y rendimiento académico en estudiantes universitarios, en la cual hallaron correlaciones altas entre motivaciónelaboración, motivación intrínseca-total estrategias; y la motivación intrínsecametacognición y autointerrogación. Asimismo encontraron una correlación significativa entre la motivación intrínseca, el valor de la tarea y las estrategias de aprendizaje.

Camarero, Buey y Herrero et al (2000) encontraron la existencia de similitud en relación con aspectos como la observación reflexiva y la conceptuación teórica en el estudiante universitario. Asimismo, se debe seguir investigando sobre el efecto de otras variables como el autoconcepto de los alumnos, motivación intrínseca y estilos de aprendizaje reflexivo-crítico.

Palomino et al (2009) investigó las relaciones entre motivación, estilos cognitivos, estrategias de aprendizaje y actividad personal en estudiantes universitarios; encontrando una correlación positiva y fuerte entre las variables motivación, estilos cognitivos, estrategias de aprendizaje y la variable actividad personal.

\section{CONCLUSIONES}

En consecuencia, aplicado los instrumentos y contrastado las hipótesis, se concluye que el nivel de motivación intrínseca alcanzado por los estudiantes de la Universidad Nacional de Ingeniería se ubica en el nivel alto y medio.

El nivel de desarrollo del estilo del aprendizaje reflexivo-crítico en los estudiantes de la Universidad Nacional de Ingeniería se ubica en el nivel alto y medio (o moderado).

Existen relaciones significativas entre el nivel de motivación intrínseca y el nivel de desarrollo del estilo de aprendizaje reflexivo-crítico de los estudiantes de la Universidad Nacional de Ingeniería.

\section{REFERENCIAS}

1. Nickerson en: Rodríguez, A. "Psicología del pensamiento". México: Limusa. pp.80, 2006.

2. Roces Montero, C., González., Nuñez., Pérez., García., Ávarez. "Las relaciones entre motivación, estrategias de aprendizaje y rendimiento académico en estudiantes universitarios". México; Universidad Autónoma de México. Informe de investigación pp.85-90, 2000.

3. Bali Chavez, G., Cázeres Castillo, A., Wisniewski. "La motivación intrínseca en el educando y evaluación de una escala para medirla”. México: Universidad Autónoma de México. Informe de investigación, 1997.

4. López García, J. "Los estilos de aprendizaje y los estilos de enseñanza". Universidad de 
Murcia: Informe de investigación pp. 179 184, 2000.

5. Camarero Suárez, F., Del Buey F., Herrero Diez, J. "Estilos y estrategias de aprendizaje en estudiantes universitarios". Universidad de Oviedo. Informe de investigación" pp. 615 622, 2000.

6. Guanipa M., Mogollón E., "Estilos de aprendizaje y estrategias cognitivas en estudiantes de Ingeniería. Maracaibo, Universidad Rafael Bellaso Chapin”. Informe de investigación pp.11 - 27, 2006.

7. Castro Gonzales, "Estilos de aprendizaje y de enseñanza de los alumnos y docentes de la Escuela Profesional de Educación". UNPRG. Lambayeque: Informe de tesis para optar el grado de maestro en Ciencias de la Educación pp. 71 - 100, 2000.

8. delgado Vásquez, E. "Relación entre estilos de aprendizaje y los estilos de pensamiento en estudiantes de maestría considerando las especialidades profesionales y el tipo de universidad. Lima. Universidad Nacional Mayor de San Marcos: Informe de tesis para optar el grado de Doctor en psicología pp. 120

9. Huauya Quispe, P. "Estilos de aprendizaje y su relación con la creatividad de estudiantes secundarios de las instituciones educativas del distrito de Ayacucho. Lima, UNEEGV. Informe de tesis para optar el grado académico de Doctor en ciencias de la Educación. pp. 96-97. 2008.

10. Palomino Orizano, J. "Estudio de las relaciones entre motivación, estilos cognitivos, estrategias de aprendizaje y actividad personal en estudiantes universitarios". Lima. UNEEGV: Informe de tesis para optar el grado académico de Doctor en Ciencias de la Educación, pp.237-238. 2009.

11. Deci., Rayan. "Intrinsic motivation and selfdetermination in human behavior". Pienum Press Nueva York, pp. 180, 1985.
12. Honey., Munford. "Using your learning styles". Maidenhead: Peter Honey pp. 72 1986.

13. Kolb, D. The lerning style inventory technical Manual. Boston: Ma McBer, 1976.

14. Valle Arias, M., Cabanach, P. "Características diferenciales de los enfoques de aprendizaje en estudiantes universitarios". Revista Psicodidáctica $\mathrm{N}^{\mathrm{o}} \quad 4 . \quad \mathrm{pp} 5$. Universidad del País Vasco, 1997.

15. Csikszenymihalyi. "Floux; The Psychology of optimal experience". Nueva York, Harper and Ron pp. 91, 1990.

16. Barnett "Higher Education: A critical bussines". Buckingham. Srhe/ Open Univeristy Press pp. 175, 1997.

17. Dewey, J. "Cómo pensamos". Nueva exposición de la relación entre pensamiento reflexivo y proceso educativo. Barcelona: Paidos. pp. 22, 1989.

18. Alonso, C. "Análisis y Diagnóstico de los Estilos de Aprendizaje en Estudiantes Universitarios". Tomo I. Madrid: Colección Tesis Doctorales. Editorial de la Universidad Complutense, 1992a.

19. Kurland, "Estilo de Aprendizaje reflexivocrítico en la Universidad". Santa Cruz, México pp. 71, 2006. Rodríguez, "Análisis y evaluación del proyecto Delphi”. Madrid. pp. 180 - 189, 2004.

20. Belenky, Golberger $\mathbf{y}$ Otros. "Women'sways of knowing y he development of self-voice and mind". Nueva York. Basic Book pp. 110, 1986.

UNI, "Memoria de la UNI". Lima. UNI, documento de trabajo. 2007.

UNI, "Memoria de la UNI". Lima UNI, documento de trabajo. 2008.

UNI, ORCE Consultado el 12 de julio del 2012. (2012). www.orce.uni.edu.pe

Correspondencia: romercen@hotmail.com

Recepción de documentos: abril 2014

Aceptación de documentos: junio 2014 